\title{
Bei COPD-Symptomen auch an Alpha-1-Antitrypsin-Mangel denken
}

\author{
Ein genetisch bedingter Mangel an Alpha-1-Antitrypsin, einem für die Lunge essenziellen \\ Proteaseinhibitor, ist mit einer rasch progredienten Verschlechterung der Lungenfunktion \\ und der Entwicklung eines Emphysems assoziiert. Da die Frühsymptome Dyspnoe und \\ Obstruktion auf eine COPD hinweisen, bleibt die Erkrankung oft lange unerkannt. Daher \\ sollten alle Patienten mit COPD spezifisch darauf getestet werden.
}

Ein Alpha-1-Antitrypsin-Mangel ist eine seltene und oftmals übersehene Unterform der COPD. In Deutschland sind Schätzungen zufolge etwa 8000 bis 16000 Patienten von einem schweren Alpha-1-Antitrypsin-Defizit betroffen; nur 10 bis $15 \%$ von ihnen sind bisher tatsächlich diagnostiziert $[1,2]$. Die Symptome Dyspnoe, Husten und Auswurf verschlechtern sich schleichend. Aufgrund dieser Symptomatik und der Seltenheit eines solchen Mangels stehe die Erkrankung im Schatten der COPD, berichtete Prof. Felix J.F. Herth, Heidelberg. Viele der Betroffenen haben bereits einen langen Leidensweg hinter sich, bis die Erkrankung nach durchschnittlich 4 bis 6 Jahren richtig diagnostiziert wird [3]. Allerdings gibt es eine Reihe wichtiger Unterschiede zwischen Alpha-1-Antitrypsin-Mangel und COPD: Wenn sich die Symptome bereits zwischen dem 30. und 40. Lebensjahr manifestieren, sollte man hellhörig werden. Zudem schreitet der Verlust der Lungenfunktion wesentlicher rascher fort als bei einer COPD.

\section{Autosomal-rezessiv vererbter Gendefekt}

Ursache des Alpha-1-Antitrypsin-Mangels ist ein autosomal-rezessiv vererbter Gendefekt auf dem langen Arm von Chromosom 14. Alpha-1-Antitrypsin wird kontinuierlich in der Leber gebildet, ins Blut freigesetzt und gelangt so in die Lunge [4]. Das aus 394 Aminosäuren bestehende Glykopeptid ist der wichtigste Proteasenhemmer in der Lunge, wo es v.a. als Gegenspieler der neutrophilen Elastase fungiert, die Fremdpartikel wie Keime, Staub und Rauchpartikel abbaut. Bei gesunden Menschen besteht ein streng kontrolliertes Gleichgewicht zwischen der Enzymaktivität von Alpha-1-Antitrypsin und den körpereigenen Proteasen. Wenn der Proteasehemmer jedoch in unzureichender Menge gebildet wird, greift dieser Regulationsmechanismus nicht mehr. Daher ist bei Patienten mit Alpha-1-AntitrypsinMangel die Kontrolle der Elastase und anderer Proteasen eingeschränkt. Die Folge ist eine fortschreitende Zerstörung der Alveolarsepten, was sich klinisch als Lungenemphysem bemerkbar macht.

\section{Neuer Schnelltest vereinfacht die Diagnose}

Einen Alpha-1-Antitrypsin-Mangel kann man nicht klinisch diagnostizieren. Alle Patienten mit COPD sowie symptomatische Asthma-Patienten mit ungenügend reversibler Atemwegsobstruktion sollten auf einen möglichen Alpha-1-Antitrypsin-Mangel getestet werden, berichtete Dr. Timm Greulich, Marburg. Gleiches gilt, wenn ein Emphysem bei jüngeren Patienten auf- tritt, eine familiäre Belastung bekannt ist, mehrere Familienmitglieder über Dyspnoe und Husten klagen oder Bronchiektasen ohne erkennbare Ursachen vorliegen. Nach den Leitlinien der Deutschen Gesellschaft für Pneumologie sollten alle COPDPatienten mindestens einmal im Leben auf einen möglichen Alpha-1-Antitrypsin-Mangel getestet werden [5]. „Die Erkrankung ist gerade bei Allgemeinmedizinern und Internisten relativ unbekannt, sodass die Empfehlungen zur Testung nicht umgesetzt werden“, bemängelte der Experte.

Der erste Schritt in der Diagnose eines Alpha-1-AntitrypsinMangels ist die quantitative Bestimmung der Konzentration des Protease-Hemmers im Serum. Wenn der Blutspiegel von Alpha1-Antitrypsin unter einem protektiven Schwellenwert liegt, erfolgt im zweiten Schritt die Phäno- und Genotypisierung. Ein neuer Schnelltest kann die Diagnose erleichtern. Dieser detektiere das veränderte Protein und erlaube eine zuverlässige Ausschlussdiagnose des in Deutschland häufigsten Alpha-1-Mutationstyps innerhalb von 15 Minuten und damit während eines Hausarztbesuches, erläuterte Greulich.

Je früher ein Alpha-1-Antitrypsin-Mangel erkannt wird, desto eher können spezifische Therapien eingeleitet werden. Die Substitution des fehlenden Alpha-1-Antitrypsins kann die Progression des Emphysems und den progredienten Rückgang der Lungenfunktion aufhalten. Die obstruktive Symptomatik wird nach den Leitlinien zur Therapie der COPD behandelt und umfasst neben dem Einsatz von Bronchodilatatoren und ggf. inhalativen Kortikosteroiden auch lebensstilmodifizierende Maßnahmen (z.B. Rauchabstinenz und Lungensport) [5]. Ultima Ratio ist eine Lungentransplantation.

Abdol A. Ameri

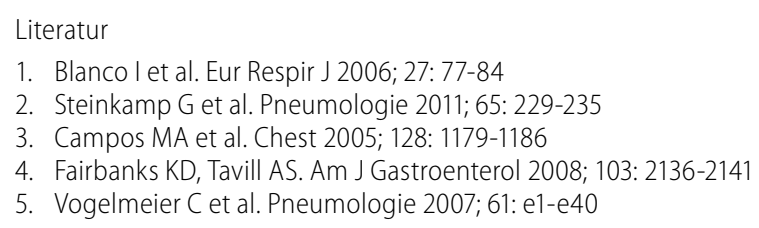

Quelle: Symposium „Seltene Atemwegserkrankungen - häufig übersehen“ am Berlin, 18. März 2015

Im Rahmen des 56. Kongress der Deutschen Gesellschaft für Pneumologie und Beatmungsmedizin, Veranstalter: CSL Behring 